\title{
High-resolution electrochemistry of the extracellular electron transfer of Escherichia coli
}

3 a, CAS Key Laboratory of Urban Pollutant Conversion, Institute of Urban Environment, Chinese

4 Academy of Sciences, Xiamen 361021, PR China;

5 b, Department of Chemistry, Technical University of Denmark, Kgs. Lyngby 2800, Denmark.

6 *Corresponding author. E-mail: yxiao@iue.ac.cn (Y.X.).

8 Supporting information for this article is available.

10 Abstract: Escherichia coli is one of the most important model bacteria in microorganism research and is

11 broadly encountered in nature. In the present study, a wild-type E. coli strain K-12 was used for 12 electrochemical investigations. Differential pulse voltammetry showed five pairs of redox peaks both for $13 \mathrm{~K}-12$ cells and the supernatant with potentials (anodic/cathodic) at $-0.450 /-0.378,-0.125 /-0.105,-0.075 /-$ $140.055,+0.192 /+0.264$, and $+0.300 /+0.414 \mathrm{~V}$ (vs. $\mathrm{Ag} / \mathrm{AgCl})$, respectively. Chronoamperometry indicates

15 that K-12 cells can produce immediate current by addition of glucose. The current production from K-12 16 can be 8 -fold enhanced by $10.0 \mu \mathrm{M}$ exogenetic vitamin $\mathrm{K} 3$, but addition of $10.0 \mu \mathrm{M}$ riboflavin did not 17 enhance the current production. Medium replacement experiments show that $50 \%$ of the K-12 biofilm 18 current was produced via direct extracellular electron transfer pathways. The study provides new insight 19 in the voltammetry of strain K-12 and confirms that $E$. coli is an electrochemically active bacterium. $E$. 20 coli has the potential to serve as a model bacterium for studying microbial extracellular electron transfer 21 mechanisms.

23 Keywords: Escherichia coli; Vitamins; Flavins; Extracellular electron transfer 


\section{Introduction}

26 Bioelectrochemical systems combine biological and electrochemical processes to generate electricity,

27 hydrogen or other useful chemicals from organic materials e.g. pollutants ${ }^{1}$. Microbial (EET), in which

28 microorganisms exchange electrons with extracellular electron donors or acceptors, is here the central

29 core process ${ }^{2-4}$. A full understanding of EET mechanisms is therefore essential to improve the production

30 of chemicals and energy as well as enhance bioremediation efficiency. Microbial EET mechanisms

31 proposed are mainly based on studies of Shewanella oneidensis or Geobacter sulfurreducens. Redox

32 proteins such as $c$-type cytochromes and other molecular electron shuttles, conductive pili/nanowires and

33 extracellular polymeric substances are regarded as the core elements in extracellular transport of electrons

$34{ }^{5,6}$. However, it is not fully clear, how microorganisms conduct the EET process, due to the limitation of

35 the model strains presently used: (i) G. sulfurreducens is an obligately anaerobic bacterium but not easy

36 to culture, with few tools available for genetic manipulation; (ii) S. oneidensis is easy to culture, but

37 genetic tools to manipulate the strain for in-depth investigations are lacking; (iii) neither of the bacteria

38 dominate the natural environment, even in bioelectrochemical systems ${ }^{7}$.

39 Escherichia coli, a facultative bacterium widely present in nature, is the most important model bacterium

40 in microbiology and molecular biology research, with clear metabolism mechanisms mapped out and

41 many available tools for genetic manipulation. However, there are many contradictory reports on EET

42 ability of $E$. coli. Schröder et al. reported that E. coli K-12 produced a current of $19.5 \mathrm{~mA}$ through

43 metabolic $\mathrm{H}_{2}$ conversion, but the electrons involved are not directly from the cells and the modified anode

44 played an important role in the bioelectricity production ${ }^{8}$. Zhang et al. recorded an enhanced current

45 production from electrochemically-evolved E. coli K-12 together with an optimized carbon/PTFE composite anode ${ }^{9}$, but did not provide a pathway for electron transfer. E. coli cells evolved under electrochemical tension showed electrochemical behavior in a microbial fuel cell with hydroquinone assumed to be the electron mediator ${ }^{10}$. However, whether the E. coli K-12 of wild type have the EET ability is still widely argued; some researchers claimed that $E$. coli is a non-exoelectrogenic bacterium 
50 since the cells could not produce current in their MFCs ${ }^{11,12}$; a small residual current was ascribed to

51 electrons and protons stored in the $E$. coli culture in a redox battery-like manner ${ }^{11}$.

52 E. coli is widely abundant in soil, and understanding of E. coli EET mechanisms, if E. coli can be

53 confirmed as an electrochemically active bacterium, can help us to illustrate and map EET processes and

54 electrochemically active microorganisms broadly ${ }^{13}$. We noted that the previous studies usually rested on

55 biofilms grown on electrodes with large surface area and high background current. Some weak but

56 important electrochemical signals may therefore have been missed. Hence, we have undertaken the

57 present study of the electrochemistry of E. coli using a high-resolution electrochemistry method,

58 differential pulse voltammetry (DPV) and the wild-type model strain K-12 with the view of obtaining

59 more details on the electrochemical profile and EET ability of E. coli.

\section{Experimental Section}

\section{Bacterial strains and growth conditions}

62 E. coli $\mathrm{K}-12$ strain was bought from the German Collection of Microorganisms and Cell Cultures (DSMZ) 63 (DSM number 498). M9 medium was used to culture $E$. coli $\mathrm{K}-12$ cells. The M9 medium ${ }^{14}$ contains (g/L)

64 glucose of $4.00, \mathrm{Na}_{2} \mathrm{HPO}_{4}$ of $6.78, \mathrm{KH}_{2} \mathrm{PO}_{4}$ of $3.00, \mathrm{NaCl}$ of $5.00, \mathrm{NH}_{4} \mathrm{Cl}$ of $1.00, \mathrm{MgSO}_{4} \cdot 7 \mathrm{H}_{2} \mathrm{O}$ of 0.493 , and $\mathrm{CaCl}_{2}$ of 0.011 . The initial $\mathrm{pH}$ of the $\mathrm{M} 9$ medium was adjusted to 7.00 using $1 \% \mathrm{HCl}$ or $1 \mathrm{M} \mathrm{NaOH}$. The strain was grown aerobically at $37{ }^{\circ} \mathrm{C}$ by shaking at $120 \mathrm{rpm}$. Glucose, $\mathrm{Na}_{2} \mathrm{HPO}_{4}, \mathrm{KH}_{2} \mathrm{PO}_{4}, \mathrm{NaCl}$, $\mathrm{NH}_{4} \mathrm{Cl}, \mathrm{MgSO}_{4} \bullet 7 \mathrm{H}_{2} \mathrm{O}$, and $\mathrm{CaCl}_{2}$ were analytically pure products from Sigma-Aldrich. Riboflavin and vitamin K3 were USP Grade from Sigma-Aldrich and from Coolaber Inc., China, respectively. E. coli K12 cells were harvested by centrifugation $\left(5000 \mathrm{~g}, 10 \mathrm{~min}, 4^{\circ} \mathrm{C}\right)$ and washed twice with $0.05 \mathrm{M}$ phosphate potassium buffer (pH 7.00) before use.

The E. coli K-12 strain grew fast aerobically in M9 medium by shaking at $125 \mathrm{rpm}$ and reached the 
73 and C) were harvested from the M9 medium for voltammetric recording by DPV, CV, and

74 chronoamperometry.

\section{Electrochemical measurements on $E$. coli $\mathrm{K}-12$ cells}

76 All electrochemical voltammetry experiments were carried out using a CHI832D or CHI732D 77 potentiostat (CHI, TX, USA) with a three-electrode chamber containing a glassy-carbon working 78 electrode (3 mm diameter), a platinum-wire counter electrode and a $\mathrm{KCl}$ saturated $\mathrm{Ag} / \mathrm{AgCl}$ reference

79 electrode ${ }^{6,15}$. All potentials reported refer to $\mathrm{Ag} / \mathrm{AgCl}$ sat. $\mathrm{KCl}$. Phosphate potassium buffer solution of $80 \quad 0.05 \mathrm{M}(\mathrm{pH} 7.00)$ was used as electrolyte. All experiments were conducted under a nitrogen atmosphere.

81 The parameters were: for cyclic voltammetry (CV), equilibrium time $5 \mathrm{~s}$, scan rate $10 \mathrm{mV} / \mathrm{s}$; for differential pulse voltammetry (DPV), equilibrium time $5 \mathrm{~s}$, increment potential $6 \mathrm{mV}$, amplitude $60 \mathrm{mV}$,

83 pulse width $0.2 \mathrm{~s}$, pulse period $0.4 \mathrm{~s}$; for $i$ - $t$ chronoamperometry, the working electrode was kept at +0.10 , $84+0.20$, or $+0.30 \mathrm{~V}$ as indicated in each figure legend.

\section{E. coli K-12 biofilm}

E. coli K-12 biofilm was anaerobically grown on carbon felt (area $1.0 \mathrm{~cm}^{2}$ ) which also served as the working electrode for subsequent $i$ - $t$ chronoamperometry. Stainless steel mesh (area $9.0 \mathrm{~cm}^{2}$ ) and a $\mathrm{KCl}$ saturated $\mathrm{Ag} / \mathrm{AgCl}$ electrode served as counter and reference electrode, respectively. The electrodes were connected to a CHI1000C potentiostat (CHI, TX, USA). The electrode system was inserted in a sealed $100 \mathrm{~mL}$ flask filled with oxygen-free medium during the cultivation. A polypropylene tube was connected to the bottom of the flask when the medium was replaced. Magnetic stirring (50 rpm) was also used.

\section{Results and Discussion}

\section{Voltammetry and chronoamperometry of $E$. coli K-12}

94 Five pairs of DPV redox peaks of $E$. coli $\mathrm{K}-12$ cells grown at $12-16$ h i.e. the early stationary growth 
and $+0.300 \mathrm{~V}$. The corresponding cathodic peak potentials were $-0.378,-0.105,-0.055,+0.264$, and $+0.414 \mathrm{~V}$. The redox peaks around $-0.400 \mathrm{~V},-0.200$ to 0 and 0.200 to $0.400 \mathrm{~V}$ were grouped into the redox peak group I, II and III, respectively (Figure 1A). The potentials of the group II and III redox peaks were so close that they sometimes overlap into a single redox peak (Figure S2). Only one or two anodic peaks were observed in the voltammetry of biofilms on electrodes with large surface area, for example $0.100,+0.100 \mathrm{~V}^{16}$ or $-0.070 \mathrm{~V}^{17}$. By conducting voltammetry on collected $E$. coli $\mathrm{K}-12$ cells on a glassy carbon electrode, the data in the present study displayed, however, a notably more sensitive E. coli electrochemical redox profile than in previous reports ${ }^{16,17}$.
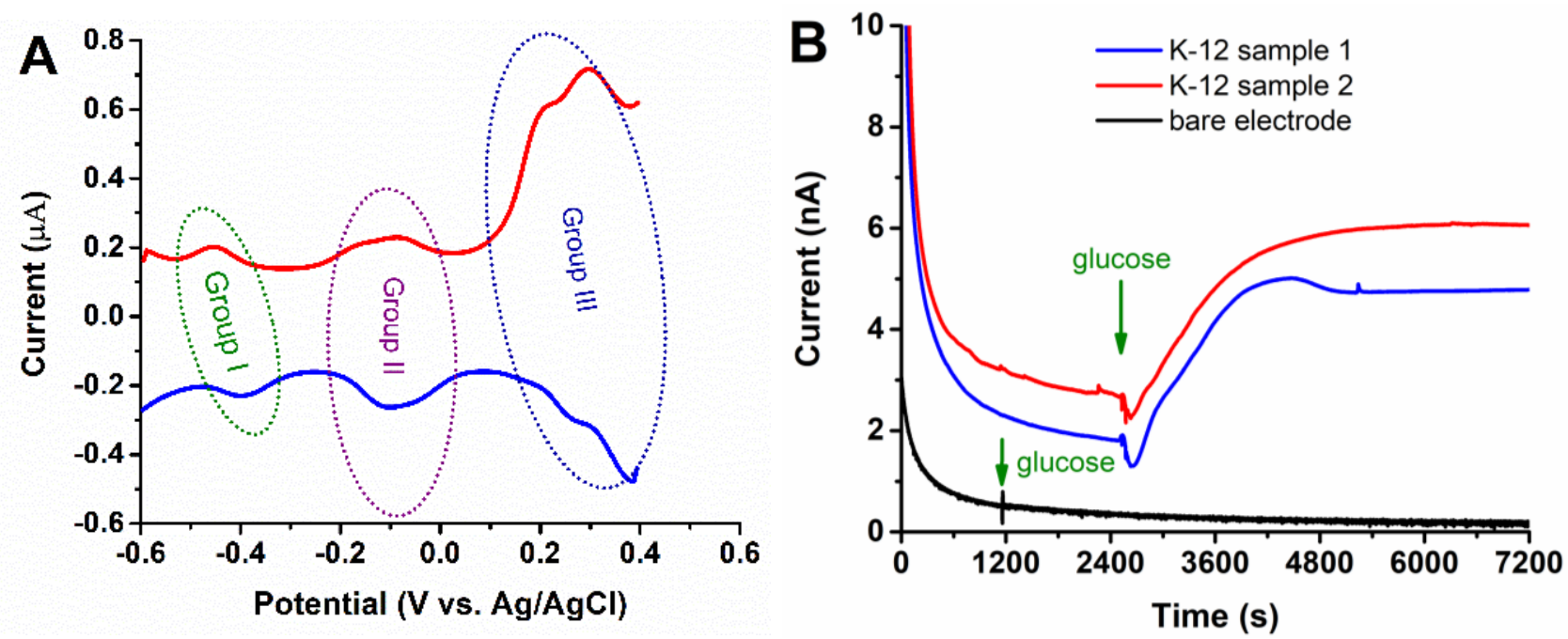

Figure 1 Electrochemical patterns of $E$. coli K-12 cells grown at 16 h. (A) DPV of $E$. coli K-12 cells dropcast onto a glassy carbon electrode. Five redox peak pairs were found and grouped into three groups. While there is only one redox peak pair in group I, group II and III each contains two pairs of redox peaks. (B) Chronoamperometry confirms a current response to added glucose from E. coli K-12 cells when the working electrode was polarized at $+0.10 \mathrm{~V} .3 .00 \mathrm{~mL} 40.0 \mathrm{~g} / \mathrm{L}$ glucose solution was added to $27.00 \mathrm{~mL}$ 110 electrolyte, making the final concentration $4.00 \mathrm{~g} / \mathrm{L}$ glucose. No current was produced from bare glassy 111 carbon electrode in $4.00 \mathrm{~g} / \mathrm{L}$ glucose. Glassy carbon, $\mathrm{Ag} / \mathrm{AgCl}$, and platinum wire served as working, 112 reference and counter electrode, respectively. In 0.05 M phosphate potassium buffer of pH 7.00.

113 All the five redox peak pairs observed for E. coli $\mathrm{K}-12$ cells were also found in the CV profiles of the 114 supernatant (Figure S3). As the scans continued, the group II current increased, whereas those in group 
115 III decreased. No obvious change was observed in group I. These results indicate that the electroactive

116 molecules on the cell membrane are also excreted to the supernatant. Furthermore, it seems that the

117 molecules related to group II are easily adsorbed on glassy carbon while those related to group III are

118 very unstable, since the peak current of this group continued to decrease.

119 Chronoamperometry confirmed that $E$. coli $\mathrm{K}-12$ cells can produce current and have EET ability using 120 glucose as electron donor at a polarization potential of $+0.10 \mathrm{~V}$, whereas glucose cannot be used for 121 current production without cells (Figure 1B). Compared to glucose, E. coli K-12 cells used lactose for a 122 smaller current production while they did not produce current from lactate and acetate $1 \mathrm{~h}$ following 123 substrate addition (Figure S4). Furthermore, biofilm formed anaerobically on carbon felt $\left(1.0 \mathrm{~cm}^{2}\right) \mathrm{can}$ 124 produce a current increment of $0.20 \mu \mathrm{A}$ by supplying glucose at a polarization potential of $+0.20 \mathrm{~V}$ 125 (Figure S5). These results confirm that E. coli is an electrochemically active bacterium, able to produce 126 electricity from appropriate organic substrate such as glucose, and redox peak groups I and II may both 127 contribute to the electricity production based on the hold potential of $+0.20 \mathrm{~V}$.

\section{What is the role of riboflavin in EET?}

129 The pair of redox peaks around $-0.40 \mathrm{~V}$ i.e. group I is ascribed to flavins as the redox potentials are similar 130 to flavins potentials in many other microorganisms such as Shewanella spp. ${ }^{6,18,19}$, Bacillus spp. $^{20,21}$, 131 Listeria monocytogenes ${ }^{22}$, Pichia stipitis ${ }^{20}$, and Pachysolen tannophilus ${ }^{23}$. Previous studies also reported 132 that E. coli can excrete flavins during growth with glucose ${ }^{24}$. Besides, we found that the redox peaks are 133 strengthened by riboflavin addition (Figure S6), meaning that the two substances have similar redox 134 potentials. 


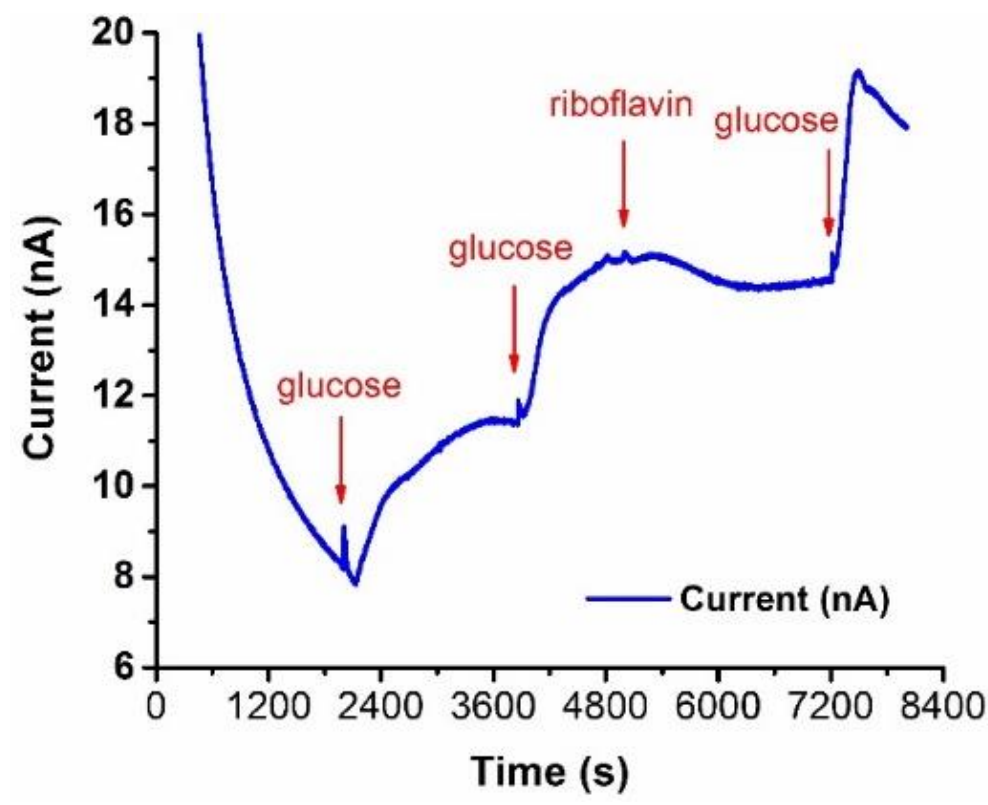

136 Figure 2 Chronoamperometry of $\boldsymbol{E}$. coli K-12 cells dropcast on glassy carbon electrode. This result

137 shows a fast current response to each glucose addition from $E$. coli K-12 cells when the working electrode 138 was polarized at $+0.20 \mathrm{~V}$. Each time glucose with a final concentration of $1.00 \mathrm{~g} / \mathrm{L}$ increase was added. 139 However, no current increment was observed after the addition of riboflavin (final $1.0 \mu \mathrm{M}$ ). $\mathrm{The} \mathrm{Ag} / \mathrm{AgCl}$ 140 and platinum wire served as reference and counter electrode, respectively. $0.05 \mathrm{M}$ phosphate buffer of $\mathrm{pH}$ 1417.0.

142 Flavins have been widely reported as ET mediators for microbial EET process ${ }^{6,20,21,23}$, but low 143 concentration of riboflavin cannot be used by E. coli $\mathrm{K}-12$ cells to facilitate its EET as no current 144 increment was observed after the addition of extra $10.0 \mu \mathrm{M}$ riboflavin (Figure 2). This result agrees well 145 with a previous study which showed that $E$. coli secreted three types of flavins ${ }^{19}$, but that E. coli did not 146 utilize these molecules in EET ${ }^{25}$. The result further confirmed that the electric current produced by $E$. 147 coli $\mathrm{K}-12$ at polarization potentials +0.10 or $+0.20 \mathrm{~V}$ are mainly contributed by the redox peak group II, 148 but not by group I ascribing to flavins.

\section{Effect of vitamin K3 on the $E$. coli EET}

150 Previous reports suggested that E. coli may use menaquinone or related compounds as mediators for EET $151{ }^{17}$, and the bacterium can definitely produce vitamin $\mathrm{K} 2$, i.e. menaquinone ${ }^{26}$. However, vitamin $\mathrm{K} 2$ is 
152 not soluble in water and it is not obvious, how E. coli can utilize this substance as an electron shuttle in

153 solution. One possible explanation if the compound is truly a $\mathrm{K} 2$ molecule, is that this molecule may

154 combine with another molecule or even proteins. In the present study, we added another soluble synthetic

155 vitamin $\mathrm{K} 3$ (final concentration $10.0 \mu \mathrm{M}$ ) with the same functional group i.e. the quinone group to test

156 whether $E$. coli $\mathrm{K}-12$ cells can use it as a mediator during the $\mathrm{CV}$ of $E$. coli $\mathrm{K}-12$. The result shows that 157 compared with the redox peak group II, vitamin K3 gave more negative redox peak potentials and 158 definitely strengthened the oxidative current but weakened the reduction current of the E. coli $\mathrm{K}-12$ strain 159 (Figure 3A).

160

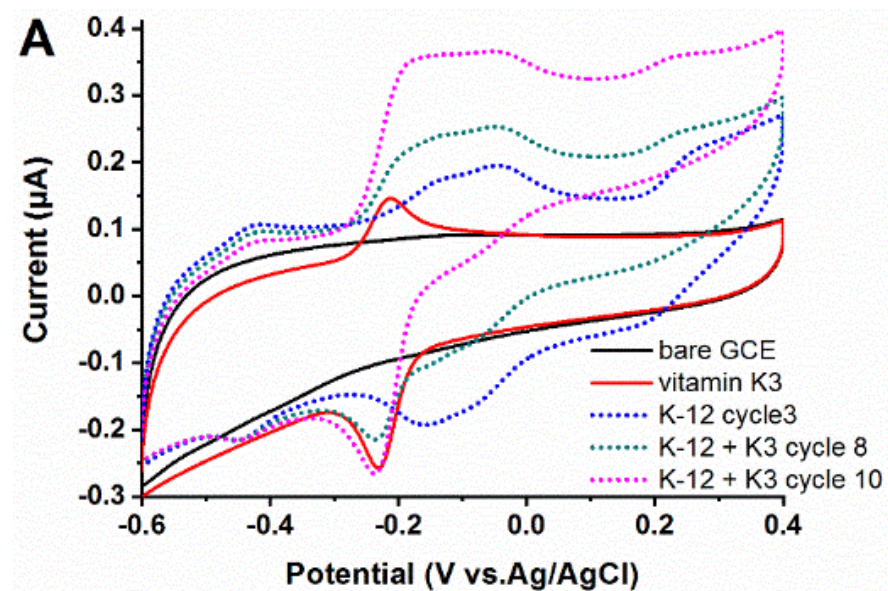

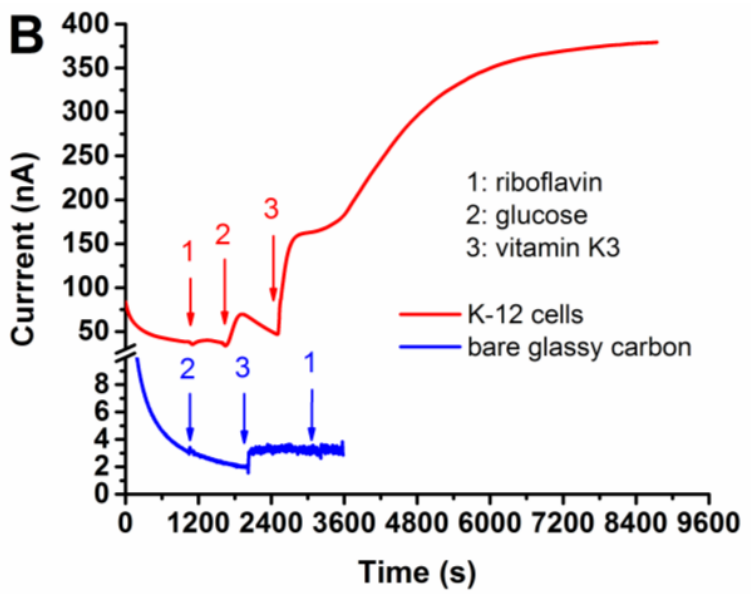

161 Figure 3 Effects of vitamin K3 on the EET of E. coli K-12. (A) CV of vitamin K3 and E. coli K-12 162 cells grown for $16 \mathrm{~h}$ with and without extra vitamin $\mathrm{K} 3$ added. $10.0 \mu \mathrm{M}$ vitamin $\mathrm{K} 3$ showed a pair of 163 redox peaks around $-0.200 \mathrm{~V}$ (red line). $\mathrm{K} 3$ (final $10.0 \mu \mathrm{M}$ ) was added to the electrolyte at the beginning 164 of cycle 7. The original CV pattern (blue dotted line, data from cycle 3) showed the three groups of redox 165 peaks from $E$. coli $\mathrm{K}-12$. The presence of $\mathrm{K} 3$ gradually strengthened the oxidative current beginning at $166 \quad 0.250 \mathrm{~V}$ (green dotted line and purple dashed line, data from cycle 8 and 10, respectively) as it diffused 167 into the biofilm on the glassy carbon surface. Scan rate $10 \mathrm{mV} / \mathrm{s}$. (B) Chronoamperometry shows that $E$. coli $\mathrm{K}-12$ cells can use vitamin K3 but not riboflavin to enhance EET. Only a very small current increment 169 from bare glassy carbon on addition of vitamin $\mathrm{K} 3$ was observed. Glassy carbon, $\mathrm{Ag} / \mathrm{AgCl}$ and platinum 170 wire served as working, reference and counter electrodes, respectively. $0.05 \mathrm{M}$ potassium phosphate 
171 buffer, $\mathrm{pH}$ 7.00. The working electrode potential was kept at $+0.30 \mathrm{~V}$. The final concentration of 172 riboflavin, glucose and vitamin $\mathrm{K} 3$ were $10.0 \mu \mathrm{M}, 4.00 \mathrm{~g} / \mathrm{L}$ and $10.0 \mu \mathrm{M}$, respectively.

173 We further evaluated the impact of vitamin $\mathrm{K} 3$ on the current production of E. coli $\mathrm{K}-12$ from glucose 174 and compared with that of riboflavin by sequentially adding riboflavin, glucose and K3 into the electrolyte 175 (Figure 3B). Riboflavin apparently had very little positive effect on the EET of E. coli K-12 cells, and we only observed a very small current increment (from 35.5 to $40.1 \mathrm{nA}$ ) caused by the addition of $10.0 \mu \mathrm{M}$ electrolyte when the current was decreasing. The current continued to increase very fast from $46.8 \mathrm{nA}$ to more than $380.0 \mathrm{nA}$. A current increment of only about $1.0 \mathrm{nA}$ was observed when the same amount of glucose and vitamin $\mathrm{K} 3$ was added to a similar system but without $E$. coli $\mathrm{K}-12$ cells on the working electrode (Figure 3B).

Vitamin K3 can also boost current from E. coli K-12 biofilm formed on carbon felt (area about $1.0 \mathrm{~cm}^{2}$,

184 Figure $\mathrm{S} 7$ ). These results indicate that E. coli $\mathrm{K}-12$ cells can use water soluble vitamin $\mathrm{K} 3$ as a mediator or combine this molecule with something on the cell surface to promote significantly the EET process and current production. However, whether E. coli can use vitamin K2 with the same quinone group as that in vitamin $\mathrm{K} 3$ to facilitate the EET process is not clear.

\section{Direct or indirect mechanisms dominate the EET process of $E$. coli K-12 cells?}

189 Following a previous study on $S$. oneidensis MR-1 ${ }^{18}$, in the present study, we have employed medium 190 replacement to identify which, direct or indirect pathway, dominates the EET process of E. coli K-12 cells. As shown in Figure S8, about 50\% oxidative current remains after the original supernatant is replaced by oxygen-free fresh M9 medium. These results were entirely reproducible both in 60 and $18 \mathrm{~h}-$ old E. coli K-12 biofilms formed on carbon felt. The results indicate that direct and indirect electron transfer mechanisms are of equal importance in the EET of $E$. coli cells. Since flavins do not contribute 
196 by the redox peak groups II and III. However, more in-depth work including molecular biology and electrochemistry are needed to specify the contribution of each redox peak to direct or indirect electron transfer.

\section{Conclusions}

200 By using small working electrodes and highly sensitive DPV measurements, high-resolution 201 voltammetric profiles of $E$. coli $\mathrm{K}-12$ were acquired in the present study. Five pairs of DPV redox peaks 202 in the electrochemical window -0.600 to $+0.400 \mathrm{~V}$ were disclosed. The redox peaks around $-0.400 \mathrm{~V}$ (the 203 group I peaks) belong to flavins which could not be utilized by $E$. coli K-12 cells to facilitate their EET 204 processes. E. coli K-12 cells can instead use water soluble vitamin K3 to enhance the oxidative current 205 from glucose. Direct and indirect electron transfer mechanisms are of equal importance in the EET of $E$. 206 coli $\mathrm{K}-12$ cells. Though wildtype $E$. coli $\mathrm{K}-12$ produces relative small currents, this study confirms $E$. 207 coli as an electrochemically active bacterium. To fully illustrate the EET process of E. coli, future 208 combined work from electrochemistry and molecular biology, can be performed to elucidate the origins 209 of the other four pairs of redox peaks. Such information would also boost the application of $E$. coli in 210 bioelectrochemical systems and bioelectricity production.

\section{Acknowledgements}

212 Financial support from the National Natural Science Foundation of China (51878640, 51478451), the

213 Youth Innovation Promotion Association of Chinese Academy of Sciences (2018344), the Carlsberg 214 Foundation (CF15-0164), China Scholarship Council ([2016]3100), Universities Denmark and Otto 215 Mønsted Foundation is gratefully appreciated.

\section{Contributions}

217 Y.X., F.Z. and J.Z. designed the experiments. Y.X., Z.Z. and H.G. performed the experiments. Y.X. 218 drafted the manuscript. All the authors revised and approved the manuscript. 
220 1. M.M. Gul, and K.S. Ahmad, Bioelectrochemical systems: sustainable bio-energy powerhouses. $221 \quad$ Biosensors Bioelectron. 142 (2019) 111576.

222 2. C. Koch, and F. Harnisch, Is there a specific ecological niche for electroactive microorganisms? ChemElectroChem 3 (2016) 1282.

224 3. B.E. Logan, R. Rossi, A.a. Ragab, and P.E. Saikaly, Electroactive microorganisms in bioelectrochemical systems. Nat. Rev. Microbiol. 17 (2019) 307-319.

226 4. S.H. Saunders, and D.K. Newman, Extracellular electron transfer transcends microbe-mineral interactions. Cell Host \& Microbe 24 (2018) 611-613.

$228 \quad 5 . \quad$ D.R. Lovely, Electromicrobiology. Annu. Rev. Microbiol. 66 (2012) 391-409.

229 6. Y. Xiao, E. Zhang, J. Zhang, Y. Dai, Z. Yang, H.E. Christensen, J. Ulstrup, et al, Extracellular polymeric substances are transient media for microbial extracellular electron transfer. Sci. Adv. $3(2017)$ e1700623.

232 7. Y. Xiao, Y. Zheng, S. Wu, E.-H. Zhang, Z. Chen, P. Liang, X. Huang, et al, Pyrosequencing reveals a core community of anodic bacterial biofilms in bioelectrochemical systems from China. Front. Microbiol. 6 (2015) 1410.

8. U. Schröder, J. Nießen, and F. Scholz, A generation of microbial fuel cells with current outputs

9. T. Zhang, C. Cui, S. Chen, X. Ai, H. Yang, P. Shen, and Z. Peng, A novel mediatorless microbial fuel cell based on direct biocatalysis of Escherichia coli. Chem. Commun. 42 (2006) 2257-2259.

10. Y. Qiao, C.M. Li, S.-J. Bao, Z. Lu, and Y. Hong, Direct electrochemistry and electrocatalytic mechanism of evolved Escherichia. Chem. Commun. (2008) 1290-1292.

242 11. M. Sugnaux, S. Mermoud, A.F. da Costa, M. Happe, and F. Fischer, Probing electron transfer with Escherichia coli: a method to examine exoelectronics in microbial fuel cell type systems. 
245 12. B.E. Logan, Essential data and techniques for conducting microbial fuel cell and other types of bioelectrochemical system experiments. ChemSusChem 5 (2012) 988-994.

13. C. Koch, and F. Harnisch, What is the essence of microbial electroactivity? Front. Microbiol. 7 (2016) 1890.

14. P. Howard-Flanders, and L. Theriot, Mutants of Escherichia coli K-12 defective in DNA repair and in genetic recombination. Genetics 53 (1966) 1137-1150.

15. X. Wu, F. Zhao, N. Rahunen, J.R. Varcoe, C. Avignone - Rossa, A.E. Thumser, and R.C. Slade,

A role for microbial palladium nanoparticles in extracellular electron transfer. Angew. Chem. Int. Ed. 123 (2011) 447-450.

16. T. Zhang, C. Cui, S. Chen, H. Yang, and P. Shen, The direct electrocatalysis of Escherichia coli

17. Y.-F. Wang, S. Tsujimura, S.-S. Cheng, and K. Kano, Self-excreted mediator from Escherichia coli K-12 for electron transfer to carbon electrodes. Appl. Microbiol. Biotechnol. 76 (2007) 1439-1446.

18. E. Marsili, D.B. Baron, I.D. Shikhare, D. Coursolle, J.A. Gralnick, and D.R. Bond, Shewanella secretes flavins that mediate extracellular electron transfer. Proc. Natl. Acad. Sci. U.S.A. 105

19. (2008) 3968-3973. 623.

20. S. Wu, Y. Xiao, L. Wang, Y. Zheng, K. Chang, Z. Zheng, Z. Yang, et al, Extracellular electron transfer mediated by flavins in gram-positive Bacillus sp. WS-XY1 and yeast Pichia stipitis. Electrochim. Acta 146 (2014) 564-567. 
269 21. L.-X. You, L.-D. Liu, Y. Xiao, Y.-F. Dai, B.-L. Chen, Y.-X. Jiang, and F. Zhao, Flavins mediate extracellular electron transfer in Gram-positive Bacillus megaterium strain LLD-1. Bioelectrochemistry 119 (2018) 196-202.

22. S.H. Light, L. Su, R. Rivera-Lugo, J.A. Cornejo, A. Louie, A.T. Iavarone, C.M. Ajo-Franklin, et al, A flavin-based extracellular electron transfer mechanism in diverse Gram-positive bacteria. Nature 562 (2018) 140-144.

23. S. Wu, Y. Xiao, P. Song, C. Wang, Z. Yang, R.C. Slade, and F. Zhao, Riboflavin-mediated extracellular electron transfer process involving Pachysolen tannophilus. Electrochim. Acta 210 (2016) $117-121$.

24. Y. Liu, M. Ding, W. Ling, Y. Yang, X. Zhou, B.-Z. Li, T. Chen, et al, A three-species microbial consortium for power generation. Energ. Environ. Sci. 10 (2017) 1600-1609.

$28025 . \quad$ M. Masuda, S. Freguia, Y.-F. Wang, S. Tsujimura, and K. Kano, Flavins contained in yeast extract are exploited for anodic electron transfer by Lactococcus lactis. Bioelectrochemistry 78 (2010) 173-175.

283 26. V. Sharma, K. Suvarna, R. Meganathan, and M. Hudspeth, Menaquinone (vitamin K2) biosynthesis: nucleotide sequence and expression of the menB gene from Escherichia coli. J. Bacteriol. 174 (1992) 5057-5062. 